\title{
Análisis clínico de la prevalencia de trastornos témporomandibulares en niños de 8 a 12 años de edad de la Escuela Diego Abad de Cepeda de la ciudad de Quito
}

Recibido: 2016/09/02. Aceptado: 2017/01/20. Publicado: 2017/01/01

\section{Carla Paquita Moreno Silva ${ }^{1}$ \\ Diego Carrillo ${ }^{2}$}

1 Universidad San Francisco de Quito, Colegio de Ciencias de la Salud, Escuela de Odontología, Clínica Odontológica, Campus Cumbayá, oficina CO 106, 115, casilla postal 17-1200-841. Quito-Ecuador.

Correo electrónico: karlet_89@hołmail.com

2 Universidad San Francisco de Quito, Colegio de Ciencias de la Salud, Escuela de Odontología, Clínica Odontológica, Campus Cumbayá, oficina CO 106, 115, casilla postal 17-1200-841. Quito-Ecuador.

Correo electrónico: diecarrillo@yahoo.com.mx 


\section{Resumen}

El presente estudio se realizó con el fin de conocer la prevalencia de trastornos témporomandibulares en niños de 8 a 12 años de la Escuela "Diego Abad de Cepeda" de la ciudad de Quito. La investigación se realizó en 228 niños que se distribuyeron en 128 mujeres y 100 hombres y bajo el método de observación se determinaron signos de trastornos témporomandibulares. Los trastornos que se evaluaron fueron dolor, limitación en apertura, deflexión, ruidos articulares y discrepancia entre relación céntrica y oclusión céntrica. El 49\% de la población mostró al menos uno de los trastornos, siendo más prevalente en el sexo femenino con el 64\%. Los trastornos más prevalentes en la población examinada fueron dolor con el $32 \%$ y ruidos articulares con el $25 \%$. Los trastornos se encontraron con más frecuencia en aquellos niños que tuvieron uno o más hábitos perniciosos, apiñamiento y maloclusiones como mordida profunda, mordida abierta y mordida cruzada. Los resultados que se obtuvieron en el presente estudio nos ayudan a confirmar que los trastornos témporomandibulares pueden afectar también a los niños y nos impulsa a los odontólogos a aprender más sobre la articulación témporomandibular para saber tratarla adecuadamente.

Palabras clave: articulación témporomandibular, trastornos témporomandibulares, hábitos perniciosos, apiñamiento, maloclusiones, clase molar, clase canina, sobremordida vertical, sobremordida horizontal.

\section{Abstract}

The aim of this study was to determine the prevalence of temporomandibular disorders in children from 8 to 12 years old of the school "Diego Abad de Cepeda" in the city of Quito. The investigation was performed in 228 children who were divided into 128 women and 100 men, and signs of temporomandibular disorders were evaluated under observation method. The disorders assessed were pain, limited opening, deflection, joint sounds and discrepancy between centric relation and centric occlusion. The $49 \%$ of people had at least one of these disorders, being more prevalent in girls with 64\%. The more prevalent disorders that people had were pain with $32 \%$ and joint sounds with $25 \%$. Disorders were found more often in those children who had harmful habits, dental crowding, and malocclusions such as open bite, deep bite and crossbite. The obtained results of this investigation help us to confirm that temporomandibular disorders can also affect to children and impulse to dentists to learn more about the temporomandibular joint to know how to treat it properly.

Key words: temporomandibular joint, temporomandibular disorders, harmful habits, dental crowding, maloclussions, molar relationship, canine relationship, overbite, overjet. 


\section{Introducción}

La articulación témporomandibular (ATM) es el área en donde se produce la conexión cráneo mandibular. Está formada por el cóndilo mandibular el cual se articula en la fosa mandibular del hueso temporal. Estos dos huesos se articulan mediante el disco articular, constituido por tejido conjuntivo colágeno denso, relativamente avascular, hialinizado y desprovisto de nervios. El disco es considerado como un hueso sin osificar y es quien permite movimientos complejos de la articulación como apertura, cierre, masticación, fonación, entre otros ${ }^{25}$. Se caracteriza porque es la única articulación que funciona de manera simultánea a diferencia de otras articulaciones del cuerpo.

En los tiempos de Hipócrates se empezaron a reconocer algunas alteraciones a nivel de la articulación témporomandibular, pero es hasta principios del año 1934 cuando se le empezó a dar importancia con una publicación de Costen, un otorrinolaringólogo el cual sugirió por primera vez que las alteraciones del estado dentario eran responsables de diversos síntomas del oído. A pesar de que las propuestas originales de Costen no tuvieron credibilidad, estas fueron precursoras para empezar estudios con relación a las disfunciones témporomandibulares ${ }^{2,3}$.

En 1982, se acuñó el término de disfunción témporomandibular para definir alteraciones vinculadas al sistema masticatorio que afectan a la articulación témporomandibular y su relación con la dentición, músculos y huesos que la componen ${ }^{4}$.

La disfunción témporomandibular (DTM) es un término que involucra diversas condiciones clínicas que afectan a la articulación témporomandibular, músculos de la masticación y estructuras asociadas. Estas condiciones clínicas usualmente se asocian con dolor auricular, muscular, ruidos articulares y limitación de la función mandibular. Es común también que una persona que padece de estos síntomas refiera dolor en el cuello, dolor unilateral de la cara y cefaleas crónicas 5 .

En las últimas dos décadas se han realizado varios estudios epidemiológicos sobre disfunción témporomandibular y se ha concluido que en la población adulta existe una alta prevalencia de signos y síntomas relacionados a ellos, en donde la mayoría son de sexo femenino. En cuanto a la población infantil, esta se ha mantenido relativamente al margen de estos estudios puesto que se ha considerado que los trastornos témporomandibulares eran ajenos a los niños 4 . Sin embargo, no se puede afirmar aquello puesto que algunas investigaciones muestran que el paciente más joven que mostró DTM tenía 5 años de edad ${ }^{6}$.

Esto nos lleva a pensar que la DTM es un problema que, así como afecta a los adultos también puede afectar a los niños, por tal motivo se realizó un estudio en niños de 8 a 12 años de edad de la Escuela "Diego Abad de Cepeda" de la ciudad de Quito para valorar la prevalencia de trastornos témporomandibulares en este grupo etario.

\section{Métodología}

El levantamiento de información para este estudio se llevó a cabo en dos fases que permitieron recolectar la información requerida. Para ello, en una primera fase se solicitó consentimiento informado de los representantes legales de los escolares y se llevó a cabo una encuesta que recopila información respecto al presente estudio.

La segunda fase se realizó en el mismo establecimiento educativo, dentro del horario previamente establecidos por las autoridades del colegio. Se realizó la historia clínica odontológica con el fin de recolectar datos que pudieran relacionarse con el estudio. Para el examen clínico el niño se sentó en una silla donde: 
Primero.- Mantuvo su mandíbula en posición intercuspídea con la boca cerrada mientras el examinador presionó ligeramente las articulaciones para registrar la existencia de dolor. Segundo.- se solicitó al niño que abra su boca para registrar la intensidad del dolor en la apertura mandibular mediante la escala análoga del dolor, donde del 0 al 2 se califica como un dolor leve; del 3 al 5 dolor moderado; y, 6 al 10 nivel elevado. Tercero.- se solicitó al niño que lentamente realice la apertura mandibular para registrar deflexión y limitación durante este movimiento. Рara registrar limitación se utilizó una regla milimetrada, determinando que una apertura limitada medirá 35 mm o menos. Se escogió esta medida de acuerdo a parámetros utilizados en investigaciones sobre trastornos témporomandibulares y gracias al apoyo bibliográfico para la realización de este estudio. Cuarto.- se valoró la presencia de ruidos articulares mediante auscultación con estetoscopio de la articulación temporomandibular. Quinto.- se procedió a realizar la manipulación de la mandíbula con la técnica bimanual de Dawson con el fin de llevarla a relación céntrica y registrar discrepancias entre esta y la oclusión céntrica. Se considera que una discrepancia entre relación céntrica y oclusión céntrica mayor a 2 milímetros puede provocar trastornos témporomandibulares.

Después de realizado el respectivo diagnóstico, se informó a su representante sobre el estado de salud oral y articular del niño mediante una notificación la cual fue entregada al mismo al término de la exploración.

Con el propósito de evitar algún tipo de sesgo, se tomaron en cuenta aquellos niños que cumplieron con los siguientes criterios de inclusión:

Niños y niñas que colaboren con la investigación comprendidos entre los 8 a 12 años de edad, que tengan los cuatro primeros molares en boca y no hayan sido tratados con aparatos de ortopedia/ortodoncia previamente. Niños a los que sus representantes hayan dado el consentimiento de participar y hayan llenado la encuesta de manera adecuada.

Una vez realizada la base de datos fue utilizado un código alfa-numérico para respetar en todo momento la individualidad y privacidad de cada participante, manteniendo estrictamente el propósito científico de este estudio.

- Primero: Se realizó una revisión de los datos inconsistentes y erróneos para obtener la muestra final de pacientes con la que se llevó acabo el estudio.

- Segundo: la base de datos creada en Excel fue codificada para permitir el ingreso de las variables adquiridas en la encuesta y su posterior análisis e interpretación.

- Tercero: se realizó un estudio de prevalencia trasversal analítico con muestra estadística no probabilística. Se realizó un análisis descriptivo en donde se obtuvo frecuencia con sus respectivos porcentajes de las variables edad y sexo, el cruce de variables se realizó entre variables de distribución etaria (edad y sexo) y variables causales (malos hábitos) correlacionándolos con los diferentes trastornos temporomandibulares permitiendo ver la relación causa efecto. Las variables codificadas como escalas serán agrupadas para trabajarlas como variables ordinales. Dentro del análisis bivarial se utilizará medidas de correlación y asociación, coeficiente de contingencia para el análisis nominal-nominal y $\mathrm{D}$ de Somers para el cruce de variables nominales- ordinales y ordinal-ordinal; con su respectiva medida de significancia p: 0.05 .

El programa que se utilizó para el análisis estadístico fue el programa STATA. 


\section{Resultados}

De los 228 niños que fueron examinados y cumplieron con los criterios de inclusión para el estudio, el 56\% (128 personas) correspondió al sexo femenino y el $44 \%$ (100 personas) correspondió al sexo masculino.

Aproximadamente el 49\% (112) de los 228 niños que fueron examinados presentaron trastornos témporomandibulares. El 29\% (66 niños) refirió tener uno de los cinco trastornos, el 13\% (30 niños) refirió tener dos trastornos, el 5\% (12 niños) tuvo tres trastornos, el 1\% (3 niños) tuvo cuatro trastornos y el $0.4 \%$ (1 niño) tuvo cinco trastornos. Fig. 1.

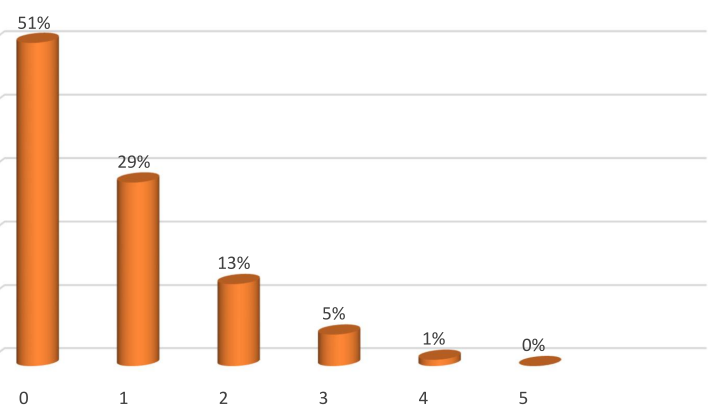

Figura 1. Prevalencia de TTM en los niños.

Del $49 \%$ de niños que presentaron trastornos témporomandibulares, el 64\% (72 personas) lo presentó el sexo femenino y el 36\% (40 personas) lo presentó el sexo masculino.

La prevalencia de trastornos témporomandibulares de acuerdo a la edad se distribuyó generalmente en niños de edades mayores (10 y 12 años), sin embargo, la población que demostró más afecciones en sus ATM fueron los niños de 8 años.

El primer trastorno que se evaluó fue el dolor, el $40 \%$ manifestó tener dolor leve, el $50 \%$ dolor moderado y el $10 \%$ dolor elevado. Fig. 2 .

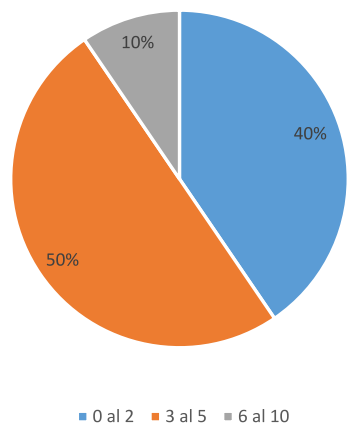

Figura 2. Escala de dolor en niños que presentaron dolor.

La deflexión mandibular que fue examinada en los 228 niños fue medida en milímetros. De esta muestra, el 12\% (28 niños) presentó deflexión al abrir su boca Fig. 4. El 53\% (15 niños) tuvo una deflexión mandibular de un milímetro, el 4\% (1 niño) tuvo una deflexión de 1.5 milímetros; el 39\% (11 niños) tuvo deflexión de 2 milímetros; y el 4\% (1 niño) tuvo deflexión de 3 milímetros. Fig. 3.

Niños con Deflexión mandibular

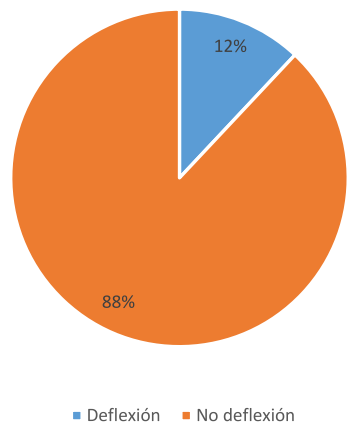

Figura 3. Niños que presentaron deflexión mandibular.

La limitación en la apertura es otro de los trastornos que se examinó, este se midió en milímetros con una regla milimetrada. Se tomó como promedio 35 milímetros para detectar una limitación; una apertura menor o igual a 35 mm se considera como limitación en apertura. 
De los 228 niños, el 3\% (7 niños) tuvo una apertura mandibular menor o igual a 35 milímetros. Fig. 4.

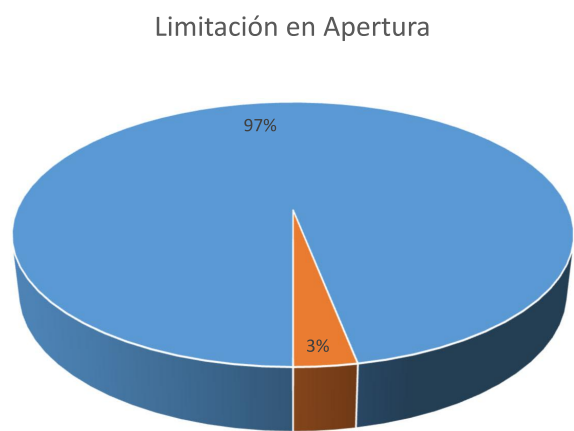

No Sí

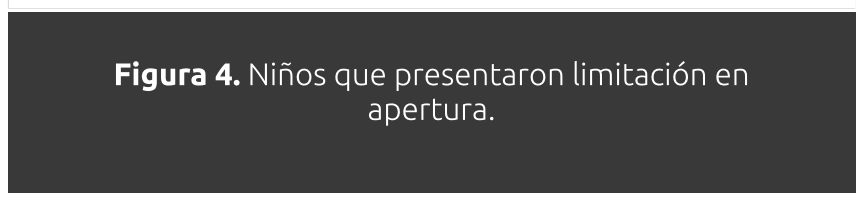

Otro trastorno que se examinó en los 228 niños fue la existencia de ruidos articulares durante la función. Este trastorno fue determinado mediante la auscultación con estetoscopio en cada una de las articulaciones.

De los 228 niños, el 25\% de ellos refirieron ruidos articulares. El 21\% tuvo ruidos en una ATM y el $4 \%$ tuvo ruidos en las dos ATM. Fig. 5.

Ruidos en ATM

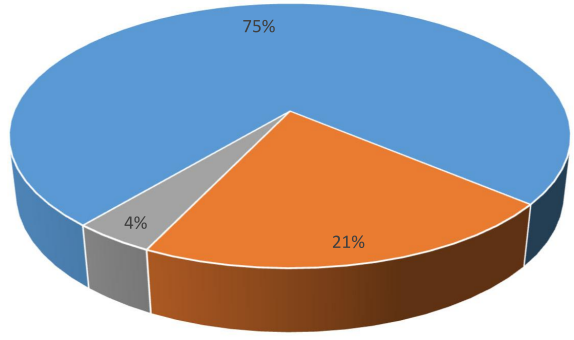

No tuvieron ruidos Una ATM Dos ATM

Figura 5. Prevalencia de ruidos articulares.

El 3\% de la población examinada tuvo una discrepancia mayor a dos milímetros, lo cual puede desencadenar un desorden témporomandibular. Fig. 6.

Discrepancia entre RC y OC

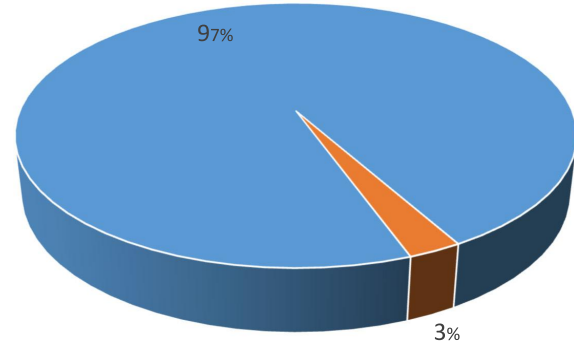

- Discrepancia menor a $2 \mathrm{~mm}$ Discrepancia mayor a $2 \mathrm{~mm}$

Figura 6. Niños que presentaron discrepancia entre RC y OC.

En este estudio también se valoró la prevalencia de trastornos témporomandibulares en cuanto a la presencia de hábitos perniciosos, traumatismos en la mandíbula y maloclusiones severas (mordida profunda, mordida cruzada y mordida abierta).

El 53\% de los niños examinados presentaron hábitos perniciosos como onicofagia, morder objetos duros, masticar chicle y succión labial, de los cuales el $38 \%$ tuvo dolor en las ATM, el $24 \%$ tuvo ruidos, el $11 \%$ tuvo deflexión, el $4 \%$ discrepancia entre OC y RC y el 3\% limitación en apertura. Fig. 7.

\section{Porcentaje de TTM en niños con hábitos}

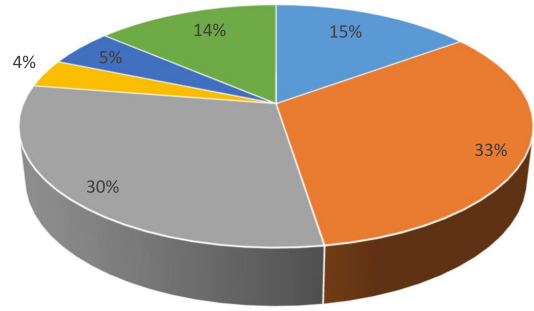

Dolor reposo Dolor función Ruidos

× Limitación en apertura Discrepancia entre RC y OC $\times$ Deflexión

Figura 7. Porcentaje de TTM en niños con hábitos perniciosos. 
Uno de los aspectos más importantes que pueden determinar un trastorno articular son los traumatismos fuertes recibidos directamente en la mandíbula. De los 228 niños que fueron examinados, el 10\% (23 niños) reportó haber recibido este tipo de traumatismos en alguna etapa de su vida. El 43\% manifestó dolor, el 25\% limitación en apertura, el $25 \%$ ruidos articulares, el $6 \%$ discrepancia entre relación céntrica y oclusión céntrica y el 3\% deflexión. Fig. 8.

TTM en traumatismo en la mandíbula

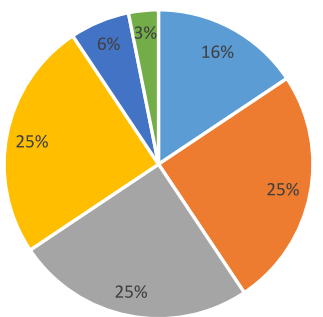

- Dolor en Reposo

- Dolor en Función $\quad$ - Limitación en Apertura

II Ruidos

- Discrepancia RC y OC = Deflexió

Figura 8. Porcentaje de TTM en niños que tuvieron traumatismos en la mandíbula.

Chi²: 0.61, p: 0.73. dS: 0.41, p: 0.51

Otro aspecto que se tomó en cuenta para la examinación, fue la determinación de maloclusiones como mordida cruzada anterior y posterior, mordida abierta anterior y posterior, y mordida profunda anterior y posterior.

De los 228 niños que se examinaron, el 36\% (83 niños) presentó las maloclusiones que se han mencionado. De este 36\%, el 49\% (41 niños) presentó signos de TTM. El 46\% presentó dolor, el $37 \%$ ruidos, el $12 \%$ deflexión, el 3\% limitación en apertura y el $2 \%$ discrepancia entre RC y OC. Fig. 9.

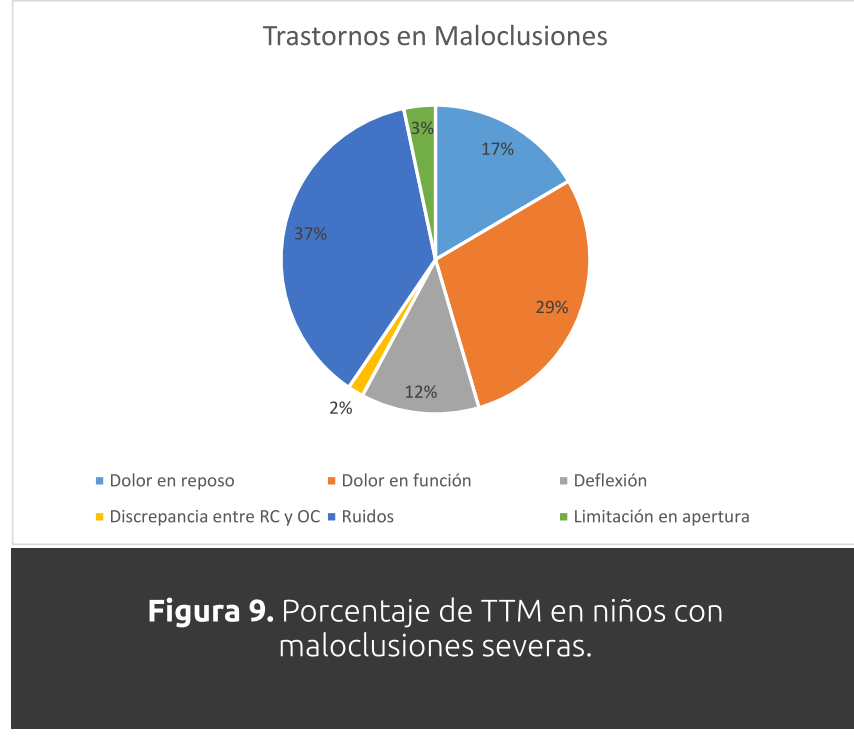

\section{Discusión}

En la presente investigación se examinaron 228 niños entre las edades de 8 a 12 años con la finalidad de establecer si existe una alta prevalencia de trastornos témporomandibulares en este grupo etario. Los signos que se evaluaron fueron dolor, ruidos, limitación en apertura, deflexión y discrepancia entre relación céntrica y oclusión céntrica. El resultado que se encontró fue que el $49 \%$ de la población examinada presentó al menos uno de los trastornos antes descritos.

Este resultado coincide con los resultados que se han obtenido en otros estudios. Por ejemplo, Nilner et al $\mathbf{l}^{\mathbf{8}}$ en 440 niños entre las edades de 7-14 años encontraron que el $36 \%$ refirió síntomas y el $64 \%$ refirió signos asociados a trastornos témporomandibulares. En otro estudio realizado por Nilner \& Lassing ${ }^{9}$ en 1988 en Suecia, en una muestra de 440 niños entre las edades de 7-14 años, el 36\% refirió síntomas y el $72 \%$ refirió signos asociados a TTM. En contraste, Martínez et al ${ }^{7}$ en Cuba, determinaron que de 600 niños examinados entre las edades de 7-11 años, sólo el $21 \%$ presentó disfunción témporomandibular.

Un aspecto importante a mencionar es que los trastornos témporomandibulares son más frecuentes en el sexo femenino que en el sexo masculino. Serrano et al ${ }^{4}$ reportaron que un 
estudio realizado por Najlla Alamoudi en 506 niños observó que el $16.53 \%$ presentó signos y síntomas de DTM y mencionó también que la prevalencia fue mayor en niñas que en niños. Wurgaft, et al. ${ }^{14}$ realizó un estudio en Brasil en 64 niños de 10 a 12 años de edad, y obtuvo que el $72 \%$ de personas que mostró TTM fueron las niñas. Kavuncu ${ }^{15}$ y Okeson ${ }^{16}$ también apoyan este dato en sus investigaciones, puesto que del mismo modo han encontrado una mayor prevalencia de TTM en la mujer que en el hombre. Estos datos concuerdan con lo que se obtuvo en esta investigación puesto que del 49\% de niños que refirieron signos de TTM, el 64\% lo presentaron las niñas y el $36 \%$ lo presentaron los niños.

Como se puede observar los porcentajes de TTM que se obtuvieron en cada investigación son variados, algunos encontraron que más de la mitad de su población examinada tuvo algún trastorno mientras que otros reportaron porcentajes menores, sin embargo, todos hallaron la presencia de trastornos témporomandibulares en niños, refutando afirmaciones que se hicieron años atrás sobre que los TTM eran ajenos a este grupo etario.

Analizando por separado cada uno de los trastornos témporomandibulares que se evaluaron para encontrar su prevalencia en la población infantil, se encontró lo siguiente:

Dolor: El dolor es uno de los trastornos más frecuentes que se presenta durante una examinación clínica. Riva, et al${ }^{\mathbf{1 0}}$ examinaron a 390 niños entre 6 y 12 años y hallaron que el 34\% de la población refirió dolor de ATM. Sánchez, et al. ${ }^{11}$ examinaron a 25 niños entre 4 y 11 años, y encontraron que el 25\% tuvo dolor de ATM. En este estudio se encontró que de los 228 niños examinados. El 32\% tuvo dolor de la ATM, lo cual indica que podría deberse a una lesión muscular, articular (ligamentos, disco) o trastornos inflamatorios.
Deflexión: La deflexión mandibular es el desplazamiento de la línea media de la mandíbula uno de los lados y se incrementa en máxima apertura. En niños no es muy frecuente encontrar este trastorno, sin embargo, se lo puede hallar en pequeños porcentajes. De los 228 niños que se examinaron, el 12\% tuvo deflexión, lo que nos indica que este porcentaje puede padecer de un desorden intracapsular asociado a hábitos perniciosos o maloclusiones.

- Limitación en apertura: una limitación de la apertura bucal puede producirse por un desorden de tipo muscular o de tipo intracapsular. Durante la examinación de los 228 niños, el 3\% demostró padecer una limitación en la apertura bucal. Corsini, et al11 en una investigación en Chile encontró que el $8 \%$ de 116 niños examinados entre 9 y 12 años, tuvo limitación durante la apertura. Riva, et al10 en Uruguay encontró que el $12 \%$ de 390 niños entre 6 y 12 años, tuvo limitación en apertura. Como se evidencia, los porcentajes de limitación durante la apertura mandibular son bajos en la población infantil, pero a pesar de ello un pequeño porcentaje sí lo padece.

- Ruidos: los ruidos en la articulación se evidenciaron en el 25\% de los 228 niños examinados. Este resultado concuerda con el estudio de Riva, et al. ${ }^{10}$ en Uruguay, de 390 niños entre 6 y 12 años, 34\% presentó ruidos en ATM. Corsini, et al. ${ }^{12}$ también encontró un alto porcentaje de ruidos articulares. De los 116 niños que examinaron entre 9 y 12 años, el 38\% manifestó ruidos. Esto sugiere la existencia de un desplazamiento discal el cual se evidencia por medio de los ruidos articulares. 
Discrepancia entre RC y OC: El movimiento de apertura de la mandíbula empieza con los cóndilos estabilizados en sus posiciones más altas en las fosas articulares, es decir en posición de Relación Céntrica (RC), que es la posición más antero superior de los cóndilos en la fosa glenoidea, apoyados en la pendiente posterior de la eminencia articular, con el disco adecuadamente interpuesto y la musculatura relajada o en estado de tono muscular. Por otro lado, la oclusión céntrica es la posición de la mandíbula que adopta con el fin de tener el mayor número de contactos dentarios posibles para realizar la masticación ${ }^{\mathbf{1 7}}$. Un desplazamiento resultante entre relación céntrica y oclusión céntrica puede alterar el equilibrio entre la forma y la función de una articulación témporomandibular puesto que puede interferir con el crecimiento condilar y su correcto desarrollo acarreando problemas de articulación en la edad adulta.

Para llevar la mandíbula a la posición de relación céntrica se pueden utilizar varias técnicas. La técnica elegida para la realización de este estudio fue la técnica bimanual de Dawson, la cual es muy utilizada para la desprogramación neuromuscular, sin embargo, presenta limitaciones puesto que se debe tener mucha colaboración por parte del paciente, él debe estar completamente relajado para ayudar al odontólogo a la manipulación de la mandíbula y poder llevarla a Relación Céntrica. Existen técnicas más efectivas y precisas que ayudan a una correcta desprogramación, pero requieren más tiempo para llevarlas a cabo. En este estudio se eligió la técnica de Dawson con el fin de ganar tiempo y colaboración de la población objeto de estudio, la cual fue de niños.

Un estudio realizado por Myers, et al. ${ }^{13}$ en 10 niños con mordidas cruzadas encontraron que en todos los casos hubo discrepancias entre RC y OC con un promedio de $3 \mathrm{~mm}$, y además otros signos de TTM. Este estudio encontró que el 3\% de la población examinada tuvo una discrepancia mayor a $2 \mathrm{~mm}$, lo que indica que en un futuro puede desarrollarse TTM.

Los trastornos témporomandibulares tienen un origen multifactorial. Los factores que predisponen a que una persona pueda desarrollar TTM son varios, entre ellos tenemos hábitos parafuncionales, factores emocionales, pérdida prematura de piezas, interferencias oclusales en el lado de balance, traumatismos, microtraumatismos, y maloclusiones como mordida abierta, mordida cruzada y mordida profunda.

\section{Hábitos parafuncionales y trastornos témporomandibulares}

Varios estudios, incluyendo el presente, han correlacionado a los hábitos parafuncionales con el desarrollo de trastornos témporomandibulares. Las publicaciones que han relacionado estas dos variables ratifican esta afirmación. Por ejemplo, Glaros ${ }^{17}$ y Kino ${ }^{18}$, refieren que los hábitos parafuncionales como masticar objetos duros y apretar los dientes favorecen la aparición de dolor muscular, de ATM y limitación en la función mandibular.

Una investigación realizada por Widmalm ${ }^{19}$ en 525 niños entre 4 y 6 años, reveló que los pacientes que presentaron TTM también presentaron hábitos como la onicofagia y succión digital. Además, reveló que el uso frecuente de chicles y el excesivo movimiento mandibular también se asocian con los ruidos articulares.

A esta afirmación se suma también el área de Odontología Integral para pacientes pediátricos de la Universidad de Buenos Aires. Los odontólogos que trabajaron en esta área en el período 2003-2007, encontraron que el 55\% de los niños que acudieron a consulta odontológica masticaba chicle, el $48 \%$ tuvieron onicofagia, el $28 \%$ mordían objetos duros y el $14 \%$ tenían el hábito de succionar el labio inferior. Además, determinaron que el $29 \%$ de los pacientes 
presentaba masticación unilateral o anterior; y se reportó que los signos y síntomas que se presentaron con más frecuencia para estos hábitos fueron dolor, ruidos y limitación en la apertura ${ }^{20}$.

El presente estudio también evaluó la prevalencia de hábitos perniciosos en los niños, y se determinó que el $25 \%$ de la población tiene el hábito de la onicofagia; el 13\% muerde objetos duros; el $27 \%$ mastica chicle; y el $7 \%$ tiene el hábito de succionar su labio inferior. Cuando se relacionaron estos hábitos con los trastornos témporomandibulares, se obtuvo que los trastornos más prevalentes fueron los ruidos articulares con el $24 \%$ y dolor en la función con el $26 \%$. Esto nos indica que los hábitos parafuncionales son factores potencialmente perjudicantes para la ATM y desencadenará trastornos témporomandibulares en la niñez o en un futuro. Es importante resaltar que también se evaluó la prevalencia del hábito de succión digital pero solamente una persona mostró tener ese hábito y no presento ningún signo de trastornos témporomandibulares.

\section{Traumatismos en la mandíbula y trastornos témporomandibulares}

Sobre los traumatismos recibidos en la mandíbula, Okeson ${ }^{16}$ dice que los traumatismos (directos o indirectos) siempre afectarán a la articulación. Un traumatismo directo en la mandíbula con la boca abierta dará lugar a un desplazamiento brusco en la fosa articular, lo que puede ocasionar un desplazamiento discal y causar síntomas como ruidos articulares. Si el traumatismo ocurre con la boca cerrada es menos nocivo para la articulación, pero de todos modos afectará a la ATM produciendo por ejemplo adherencias.

Serrano et al4 en su investigación en 50 niños en la Baja California, no reportó sujetos que hayan padecido de traumatismos en la mandíbula, sin embargo, recomienda que en el caso de quienes hayan sufrido este tipo de trauma se debe procurar limitar la progresión de la afectación con tratamientos para la ATM como férulas oclusales para niños, con lo cual podrá alcanzar niveles de adaptación y tolerancia sin que se produzca un ulterior trastorno mayor ${ }^{21}$.

En la población examinada, el 10\% de los niños reportó haber sufrido de un traumatismo en su mandíbula, del cual el $61 \%$ reportó signos de TTM. Los signos que más sobresalieron en estos niños fueron los ruidos (25\%), dolor (25\%) y limitación en la apertura (25\%), lo cual concuerda con lo antes mencionado por Okeson.

Del $10 \%$ de niños que reportaron haber padecido de un traumatismo en la mandíbula, el 1\% (3 niños) recibieron tratamiento para aliviar el golpe, (ningún caso reportó qué tipo de tratamiento recibieron); sin embargo, a pesar de que se trataron, el 67\% (2 niños) presentó signos de TTM como fueron ruidos (50\%) y discrepancia entre relación céntrica y oclusión céntrica (50\%), lo cual nos lleva a pensar que en realidad hubo un daño articular.

\section{Maloclusiones y trastornos témporomandibulares}

La maloclusión según Angle es la perversión del crecimiento y desarrollo normal de la dentadura. Angle clasifica las maloclusiones basándose en la hipótesis de que el primer molar y canino son los dientes más estables de la dentición y la referencia de la oclusión ${ }^{\mathbf{1 7}}$.

Clase I.- cada diente mandibular ocluye con su antagonista correspondiente y con el diente mesial adyacente. Hablando de maloclusión, se puede encontrar apiñamientos anteriores en la clase $\mathbf{~ I 7 . ~}^{17}$.

Clase II.- cuando los primeros molares inferiores ocluyen distalmente con relación a los primeros molares superiores aproximadamente igual a la anchura mesiodistal de un premolar ${ }^{17}$.

Clase III.- los primeros molares inferiores contactan mesialmente con relación a los 
primeros molares superiores, aproximadamente la anchura de un premolar ${ }^{17}$.

La relación de las maloclusiones y TTM han sido sujeto de mucha controversia. No se puede dar por hecho que tengan una correlación, sin embargo, pueden ser factores predisponentes las maloclusiones severas como la mordida abierta, mordida profunda y mordida cruzada. Las maloclusiones provocan una inestabilidad de las articulaciones puesto que existen contactos dentarios inadecuados lo que puede producir un movimiento inusual o perjudicial en la articulación en un intento de ganar estabilidad. Este desplazamiento es con frecuencia un deslizamiento transitorio entre el disco y el cóndilo, lo cual si no se corrige a tiempo mediante la ortodoncia se podrían provocar trastornos témporomandibulares.

Olsson y Lindqvist ${ }^{22}$ encontraron que los pacientes con mayores requerimientos de ortodoncia presentan signos y síntomas de TTM. Tanne et al23 descubrieron que los pacientes con mayor prevalencia de TTM fueron aquellos que tuvieron mordida abierta, mordida profunda y mordida cruzada. Wlliamson ${ }^{24}$ reportó que el $72 \%$ de pacientes ortodónticos con signos y síntomas de TTM tuvieron mordida profunda o mordida abierta.

El presente estudio encontró que el $36 \%$ de los niños examinados presentaron maloclusiones severas (mordida cruzada, mordida abierta, mordida profunda). De este porcentaje el 49\% manifestó trastornos témporomandibulares, por lo que se puede corroborar las afirmaciones realizadas en otras investigaciones.

Como se puede apreciar, las estadísticas que se obtuvieron en esta investigación coinciden en su mayoría con investigaciones realizadas por autores reconocidos. Sin embargo, también existen discrepancias con otros autores lo que confirma que el origen de los trastornos témporomandibulares es multifactorial y los resultados varían entre una y otra persona, sobre todo en los niños puesto que al estar en crecimiento continuo su articulación puede adaptarse a distintas situaciones.

\section{Conclusiones}

Con el análisis de los resultados que se obtuvieron en la examinación clínica que se realizó en los 228 estudiantes de la Escuela Diego Abad de Cepeda entre las edades de 8 a 12 años se puede concluir que:

Los trastornos témporomandibulares se presentan en ambos sexos, sin embargo, existe una frecuencia mayor en mujeres (64\%) que en hombres (36\%). Chi²: 4.75, P: 0.093. CC: 0.14 , $P: 0.093$

Los trastornos que más se manifestaron en los niños fueron ruidos articulares (25\%) y dolor (32\%).

Las edades que más trastornos manifestaron fueron los niños de 8 años con el 63\%, niños de 12 años con el $50 \%$ y niños de 10 años con el $48 \%$.

La prevalencia de dolor en las articulaciones fue más frecuente durante la función. El $21 \%$ de la población examinada manifestó dolor durante la apertura y cierre de la mandíbula. La escala de dolor que más se repitió fue un nivel moderado de dolor, sin embargo, este trastorno no resultó incapacitante para ningún paciente.

Durante la auscultación de las ATM de los niños, se detectó que el $25 \%$ tuvo ruidos articulares. El $21 \%$ tuvo ruidos en una articulación y el $4 \%$ tuvo ruidos en las dos articulaciones.

La prevalencia de desviación de la línea media de la mandíbula durante la apertura mandibular se manifestó en el $12 \%$ de la población examinada. Una deflexión mandibular de un milímetro fue la más frecuente, la cual se manifestó en el 54\% del total de la población que presentó deflexión.

La prevalencia de limitación en apertura en el presente estudio no fue alta, solamente el $3 \%$ 
tuvo una apertura menor o igual a 35 milímetros que fue el promedio determinado para evaluar este trastorno.

Se considera que una discrepancia entre relación céntrica y oclusión céntrica mayor a 2 milímetros puede provocar trastornos témporomandibulares. En este estudio se presentó una discrepancia mayor a 2 milímetros en el 3\% de la población examinada.

Los traumatismos directos en la mandíbula son grandes precursores para que se desarrolle un trastorno témporomandibular. Del 10\% que reportó haberlo tenido, el 61\% presentó signos de TTM.

Los hábitos perniciosos son factores predisponentes para que una persona desarrolle trastornos témporomandibulares. Del 53\% que presentó hábitos, el 51\% presentó trastornos témporomandibulares. Los trastornos que más se repitieron fueron dolor en la función (26\%) y ruidos (24\%).

De los 228 niños que se examinaron, el 36\% presentó maloclusiones como mordida abierta, mordida cruzada y mordida profunda. De este $36 \%$, el 49\% presentó signos de TTM. Esto nos indica que la presencia de maloclusiones predispone a una persona a desarrollar trastornos témporomandibulares.

\section{Referencias Bibliográficas}

1. Raspall Guillermo. Cirugía Maxilofacial. Madrid, España. Segunda edición. Editorial Médica Panamericana. 2001.

2. Román Toni. Historia de la disfunción témporomandibular. 2010. Disponible en http://disfunciontemporomandibulartoni.blogspot.com/2010/10/historia-delos-trastornos.html

3. Casanova J., Jiménez G y Gutiérrez. Prevalencia y factores de riesgo asociados a desórdenes témporomandibulares en una población universitaria de Campeche, México en Revista ADM: Asociación Dental Mexicana. 1998; (6): 261-265.

4. Serrano A. Fregoso C. Jiménez F. Ocampo F. Frecuencia de la disfunción témporomandibular en niños. Odontología clínica. 2009;3 (2): 4-8.

5. Gutiérrez J. Radiología e imágenes diagnósticas. Barcelona, España. Segunda edición. Corporación para investigaciones biológicas. 2000.

6. Navarro C. Cirugía oral. Madrid, España. Primera edición. ARÁN. 2008.

7. Martínez I. Disfunción témporomandibular en población de 7 - 25 y más años de edad. Municipio Matanzas. Revista Médica Electrónica. Matanzas, Cuba. 2006;28 (6).

8. Nilner $M$. Prevalence of functional disturbances and diseases of the stomatognathic system in 7-14 year olds. En Swed DentJ. 1983; (5): 189-197.

9. Nilner $M$, Lassing $S$. Prevalence of functional disturbances and diseases of the stomatognathic system in 7-14 year olds. En Swed Dent J. 1981. (5): 173-187.

10. Riva R, Sanguintti M, Rodríguez A, Guzzetti $\mathrm{L}$, Lorenzo $\mathrm{S}$, Álvarez $\mathrm{R}$, Massa $\mathrm{F}$. Prevalencia de trastornos témporo mandibulares y bruxismo en Uruguay en Odontoestomatología Scielo Uruguay. Montevideo, Uruguay. 2011; 13 (17): 5471.

11. Sánchez J, ATM y su relación con el tipo de oclusión dental, en un grupo de jóvenes mexicanos. Revista ADM. 2011; 68 (5): 237 243. 
12. Corsini, G. Determinación de los Signos y Síntomas de los Trastornos Temporomandibulares, en estudiantes de 9 a 12 años de un colegio de la comuna de Temuco, Chile. Int. J. Morphol. 2005;23 (4): 345-352.

13. Myers D, Barenie J, Bel R, Williamson E. Condylar position in children with functional posterior crossbites: before and after crossbite correction, Pediatr Dent. 1980;2:190-194.

14. Wurgaft R, Wong R. Temporomandibular Joint Remodeling for the Treatment of Temporomandibular Joint Disorders - A Clinical Case Study. 2009;2:43-49

15. Kavuncu V, Sezai S, Ayhan K. The role of systemic hypermobility and condylar hypermobility in temporomandibular joint dysfunction syndrome. Rheumatol Int. 2006;26:257-260.

16. Okeson J. Tratamiento de oclusión y afecciones témporomandibulares.

Barcelona, España. Sexta edición. ELSEVIER. 2008.

17. Glaros A, Williams K, Lausten L. The role of parafunctions, emotions and stress in predicting facial pain. J Am Dent Assoc. 2005;136 (7): 858-60.

18. Kino K. The comparison between pains, difficulties in function, and associating factors of patients in subtypes of temporomandibular disorders. J Oral Rehabil. 2005;32 (5): 315-25.

19. Widmalm S, Christiansen R, y Gunn S. Oral parafunctions as temporomandibular disorder risk factors in children. Revista Paulista de Pediatría. 1995; (13): 244-246.
20. Cortese S y Biondi A. Relación de disfunciones y hábitos parafuncionales orales con trastornos témporomandibulares en niños y adolescentes. Archivo Argentino Pediátrico. Universidad de Buenos Aires, Argentina. 2009;107 (2): 134-138.

21. Okeson J. Temporomandibular disorders in children en The American Academy of Pediatric Dentistry. 1989;11 (4): 325-327.

22. Olsson $M$, Lindqvist $B$. Mandibular function before and after orthodontic treatment. European Journal of Orthodontics. 1995; (17): 205-214.

23. Tanne K, Tanaka E, Sakuda M. Association between maloclussion and témporomandibular disorders in orthodontic patients before treatment. Journal of Orofacial Pain. 1993; (7): 156162.

24. Williamson E. Temporomandibular dysfunction in pretreatment adolescent patients. American Journal of Orthodontics. 1977; (29): 433.

25. Nelson y Ash. Wheeler Anatomía, fisiología y oclusión dental. Barcelona, España. Novena edición. ELSEVIER. 2010. 